\title{
Flip-Flopping, Primary Visibility, and the Selection of Candidates
}

\author{
By MARINA AgRANOV*
}

\begin{abstract}
We present an incomplete information model of two-stage elections in which candidates can choose different platforms in primaries and general elections. Voters do not directly observe the chosen platforms, but infer the candidates' ideologies from observing candidates' campaigns. The ability of voters to detect candidates' types depends on the visibility of the race. This model captures two patterns: the post-primary moderation effect, in which candidates pander to the party base during the primary and shift to the center in the general election; and the divisive-primary effect, which refers to the detrimental effect of hard-fought primaries on a party's generalelection prospects. (JEL D11, D72, D83)
\end{abstract}

\begin{abstract}
olitical primaries, an influential institution in the American political process, require candidates to obtain a party nomination by vote in order to compete in the general election. Two established facts about primaries are that candidates tend to pander to the party base during primaries and moderate their platforms after securing the nomination; ${ }^{1}$ and more prominent and, thus, hard-fought primaries can influence a party's chances of winning the election. ${ }^{2}$ The first observation, "post-primary moderation," follows from the premise that primary voters hold more extreme political views than the general-election voters. The second observation, the so-called "divisive-primary" hypothesis, suggests that a candidate's prospects in a general election may be affected by the visibility of the primary race.
\end{abstract}

\footnotetext{
* California Institute of Technology, 1200 E. California Boulevard, Pasadena, CA 91125 (e-mail: magranov@ hss.caltech.edu). I would like to thank Andrew Schotter and Alessandro Lizzeri for the invaluable help and encouragement they have provided during the course of this project. I thank Alex Agranov for suggesting the idea for this paper. I also thank Matt Elliott, Elliott Ash, Mark Dean, Matthew Embrey, Guillaume Frechette, Alessandro Gavazza, Anna Ingster, Daniel Martin, Rebecca Morton, Nicola Persico, Pietro Ortoleva, Debraj Ray, Ofer Setty, Chloe Tergiman, Emanuel Vespa, and seminar participants at New York University for their useful comments.

Go to http://dx.doi.org/10.1257/mic.20130036 to visit the article page for additional materials and author disclosure statement(s) or to comment in the online discussion forum.

${ }^{1}$ Using US congressional data, Burden (2001) shows that candidates adopt more extreme positions in primaries than in general elections.

${ }^{2}$ The conventional wisdom that hotly contested primaries can damage a party's chances in the general election is based on the theoretical work of Key (1952). Empirical literature that studies this conjecture has produced mixed results: Abramowitz (1988); Bernstein (1977); and Lengle, Owen, and Sonner (1995) find that prominent primaries hurt candidates in the general elections. Alvarez, Canon, and Sellers (1995) and Westlye (1991) find that prominent primaries help candidates in the general election. Atkenson (1998) and Kenney (1988) find that general election prospects are not affected by the primary visibility. Finally, Born (1981) and Hogan (2003) find a mixed relationship. In this paper, we use theoretical analysis to shed light on the relationship between the visibility of the nomination process and general election outcomes. The mechanism studied here delivers a negative correlation; i.e., it shows that prominent primaries are detrimental to a party's chances of winning general elections.
} 
These two observations hardly seem surprising. Despite this, the theoretical literature lacks a model that can deliver both of these results simultaneously. The reason is that most existing models use one of two extreme assumptions: either that candidates make binding commitments to electoral platforms (as in Downs 1957 and Hotelling 1929), ${ }^{3}$ or that announcements made by candidates are purely cheap talk (as in Alesina 1988). If a candidate commits to a platform, then the mere fact of commitment precludes moderation. If a candidate has no access to a commitment technology, then his general-election prospects should not be affected by the visibility of the primary race. Thus, a model with either of these two assumptions cannot explain both the post-primary moderation and the divisive-primary effect.

In this paper, we develop a model of two-stage electoral competition that captures both the post-primary shift and the divisive-primary effect. In our model, candidates have policy preferences and a partial commitment to these policies, which is captured by incorporating costs of lying as well as by having the candidates' platforms revealed imperfectly. Voters are forward-looking and take into account that a more extreme candidate has a smaller chance of winning the general election than a moderate one does. However, voters do not observe candidates' true ideological positions (types) and try to infer them from platforms candidates campaign on. Voters' ability to detect candidates' types are affected by the properties of the scrutiny function and summarized by prominence or visibility of a race, which measures the informativeness of a race. The more prominent the race is, the more likely voters are to learn the true type of a candidate. The candidates strategically choose campaign platforms depending on their levels of visibility, and, as a result, candidates' true preferences are partially revealed.

In equilibrium, candidates "flip-flop" by pandering to the median voter of the primary race during the primary and then shifting to the center once the nomination is obtained. In the primary, voters elect a candidate they believe to be more extreme. The extent to which candidates mimic each other depends on the costs of lying and the visibility of each stage. We show that in this equilibrium an increase in the primary visibility lowers the chances of the party holding it to win the general election. This is because prominent primaries increase the chances of moderate candidates to lose the nomination and decrease the chances of extreme challengers to win the general election.

The basic model studied in this paper treats the visibility of a race as an exogenous parameter and assumes that all voters (even those who do not participate in the primary election) observe primary campaigns. In Section IV, we consider two extensions of this basic model in which we relax each of these assumptions. In the first extension we investigate robustness of pandering behavior of candidates to the changes in the information structure. In particular, we consider the election game, in which voters who do not take part in the primary election are ignorant about events that have transpired during primary campaigns, and, therefore, hold beliefs that are

\footnotetext{
${ }^{3}$ See also Wittman (1983) who studies a one-stage election model with policy-motivated candidates and Coleman (1972) who investigates a two-stage election model with office-motivated candidates. The main assumption in this class of models is that candidates choose one position, at the beginning of the election, and this position will be implemented if they get elected.
} 
different from voters who take part in the primary election. We show that pandering equilibrium is robust to this change in the information structure and flip-flopping equilibrium exists in the election game with partially informative primaries. Our second extension explores how primary visibility is determined. Here we consider a model in which candidates can influence primary visibility via costly investment that precedes the primary race. We show that in this setup, there exists a separating equilibrium in which extreme candidates invest in boosting primary visibility, while moderate ones refrain from doing so.

Our model belongs to the class of signal-jamming models, as coined by Fudenberg and Tirole (1986). In both election stages, some types of politicians try not to signal information about themselves by generating a jammed signal, which interferes with the inference problem faced by voters. In the primaries, it is moderate politicians who prefer to conceal their type from the voters, while in the general election it is politicians with more extreme views. The reason politicians engage in signal jamming is the trade-off that candidates face: the probability of winning versus the policy outcome should you win. This trade-off is the classical one in political economy and has been explored by Downs (1957), Wittman (1983), Calvert (1985), and many other models. The difference in this paper is that this trade-off is being made by the median voter of the primary election rather than the candidate herself. To execute the trade-off, the primary median has to learn the type of candidate he is nominating. This selection problem itself induces a trade-off: the primary median wants to nominate a more extreme type (which is closer to his policy preferences) but as he learns whether a candidate is extreme or not, so too does the general election median. This lowers the probability further that an extreme nominee will win the general election both directly and indirectly. The indirect effect is that a candidate that is strongly perceived to be extreme will pander less to the general election median.

The rest of the paper is structured as follows. We survey the related literature at the end of this section. Section I lays out the model. In Section II we characterize the pandering equilibrium. In Section III we obtain comparative statics results. Section IV presents two extensions of the basic model, and, finally, in Section V we offer some conclusions.

Related Literature.-Our model belongs to the literature that studies information transmission in elections. This literature is vast, and can be broadly divided into two classes of models. The first class includes voting games with incomplete information, in which voters attempt to signal information to the candidates in order to influence policy choices (see Piketty 2000, Razin 2003, Meirowitz 2005, Shotts 2006, and Meirowitz and Shotts 2009). Within this class of models, the paper which is most closely related to ours is that of Meirowitz (2005) who studies two-stage elections, in which candidates' policy preferences are common knowledge while the distribution of voters' ideal points is uncertain at the time of the primary race. As a consequence, all candidates running in the primary election prefer to remain vague and not to commit to a policy platform because when they do, they become vulnerable in the general election stage and are likely to be defeated by a candidate from the opposing party. This happens because the opposing candidate in this case is able to choose the winning platform since platforms chosen during the primary 
race are known and cannot be altered at this stage; and the distribution of the voters' ideal points is known with certainty at this moment. $\square^{4}$

The second class of models, the current model included, considers voting games in which candidates signal their policy preferences to the voters to influence their chances of winning the elections. In particular, Banks (1990) shows that when misrepresenting one's true ideological position (lying) is costly, extreme candidates tend to reveal their true positions while moderate ones tend to pool together. Callander and Wilkie (2007) extend Banks's model to allow for heterogeneous costs of lying and find that, although liars are favored in the elections, the honest types are not always defeated. Kartik and McAfee (2007) study a related model, in which a fraction of candidates have a "character" and are exogenously committed to campaign platforms. In a recent study, Kartik and Van Weelden (2015) consider an election model with nonbinding communication and show that under suitable conditions cheap-talk electoral announcements can be informative. The pandering behavior of politicians in this model is driven by reputation concerns, and it increases in voters' uncertainty about politician type. The model presented in this paper departs from the above models in that we study two-stage elections, in which candidates face electorates with different preferences in the primary and in the general election stages; and candidates can choose different platforms in each election stage..$^{5}$ These crucial differences raise the question of how much information can we expect to be revealed in this two-stage election process.

Our model also belongs to the literature that investigates the effects of primary elections on candidates' and voters' behavior. Coleman (1972) and Owen and Grofman (2006) discuss the polarizing effect of primaries when candidates are constrained to offer the same platform in the general election as they had stated in the primary. Adams and Merrill (2008) demonstrate that candidates who have stronger campaign abilities are more likely to get elected in primaries. Several papers, including Dekel and Piccione (2000, 2014), Battaglini (2005), Callander (2007), and Ali and Kartik (2012), explore sequential nature of the primary elections and investigate momentum effects that may arise in these environments. ${ }^{6}$ Hirano, Snyder, and Ting

\footnotetext{
${ }^{4}$ See also, Alesina and Holden (2008) for a model of electoral competition with campaign contributions, in which candidates announce a range of policy preferences rather than a single point in attempt to balance median voter preferences and those of campaign contributors.

${ }^{5}$ Kartik and McAfee (2007) consider an extension of the one-stage model, in which candidates who compete in the general election are selected by the primary election. Similar to my setup, in this model, a candidate must appeal to electorates with different preferences during the primary and during the general election. However, candidates are not allowed to adjust their platforms after the primary election. This ability to adjust platforms turns out to be an important feature of my model that generates flip-flopping behavior in equilibrium and produces a number of interesting predictions that I explore.

${ }^{6}$ Dekel and Piccione (2000) consider sequential elections with two alternatives and show that informative symmetric equilibria of a simultaneous voting game are equilibria in any sequential voting game as well. Battaglini (2005) shows that results of Dekel and Piccione (2000) are sensitive to the introduction of voting costs and possibility of abstention. Ali and Kartik (2012) construct history-dependent equilibria that exhibit momentum effects, in which voters learn from the actions taken by previous voters. In this equilibrium, the authors show that even if voters are strategic, it is optimal for each voter to play history dependent strategies if other voters do so. In other words, the symmetry underlying Dekel and Piccione (2000) result is broken because if all voters condition on history then early votes are more informative than later votes. Callander (2007) introduces a behavioral assumption and shows the existence of bandwagons when the number of voters is infinite and voters have a desire to conform with the majority. Finally, Dekel and Piccione (2014) study sequential elections in which voters can choose when they cast their vote and explore how the timing of voting depends on voters' preferences.
} 
(2009) study a model of distributive politics and show that when the nominee of the party is elected through a primary election, core voters receive positive transfers, whereas they receive nothing when only the general election matters. This result is reminiscent of pandering behavior of candidates we characterize in the current paper, albeit very different setup and research questions. 7

The most closely related paper to ours is that of Hummel (2010). Both the current model and that of Hummel (2010) consider two-stage elections with forward-looking voters and both characterize an equilibrium, in which candidates pander towards the median of the primary to obtain the nomination and then shift to the center towards the position of the population median to increase their chances of winning in the general-election stage. However, the mechanisms behind flip-flopping behavior of candidates are very different. In Hummel (2010), voters dislike when candidates run on different platforms in the primary and in the general election because the mere fact of changing platforms indicates candidates' valence characteristics. On the contrary, the mechanism proposed in this paper explores the asymmetry of information between candidates and voters: candidates know their policy preferences, while voters try to infer those from the campaign, and their ability to do so depends on the campaign visibility.

Finally, we note that to our knowledge this is the first paper that explores the divisive-primary hypothesis in a purely informational context. Furthermore, the current model provides a unified framework that accounts for flip-flopping behavior of candidates and the divisive-primary effect in the same setup and shows that both effects originate from the trade-offs related to the timing of information transmission.

\section{Election Game}

In this section, we describe the primitives of our game. We start by outlining the timing, preferences and solution concept and then talk about the assumptions. The discussion of modeling choices and interpretations is deferred to the end of the section.

The players in the election game are two candidates from the Democratic party, $k=A, B$, and a continuum of voters. For a candidate $k$, winning office requires defeating the other candidate in the primary election (stage 1) and then defeating Republican incumbent in the general election (stage 2). The timing of the game is as follows:

Stage 0-Information Stage

- Nature independently draws the types of Democratic candidates $\left(t^{A}, t^{B}\right)$ $\in\{L, M\}^{2}$, where $L$ stands for Liberal and $M$ stands for Moderate. Each candidate is $L$ with probability $\frac{1}{2}$. Types are privately observed.

- There is a continuum of voters. A voter $j$ is characterized by an ideal policy $z_{j} \in[0,1]$. The subset of voters, those with ideal points $z_{j} \in[0, \bar{z}]$, are registered Democrats.

\footnotetext{
${ }^{7}$ There are many noticeable differences between the current model and that of Hirano, Snyder, and Ting, including the main question under investigation. We focus on the information transmission and the selection of candidates in the two-stage elections, while Hirano, Snyder, and Ting study how the existence of primary elections affects the distribution of public resources.
} 
- Nature determines locations of voters. In particular, the location of the median voter $m \in[0,1]$ is drawn from a cumulative distribution function (CDF) $F$. The location of the median Democrat $d \in[0, \bar{z}]$ is drawn from a CDF $G$.

Stage 1-Primary Election

- Each candidate $k$ chooses an effort level $e_{1}^{k} \in[0,1]$ for the primary campaign.

- Signals $\left(s_{1}^{A}, s_{1}^{B}\right) \in\{\lambda, \mu\}^{2}$ regarding candidates' types are generated for the two candidates, where $\lambda$ stands for liberal and $\mu$ for moderate signal.

- All voters observe $\left(s_{1}^{A}, s_{1}^{B}\right)$.

- Registered Democrats cast their vote for one of the candidates.

- The winner of the primary is the candidate with the most votes. This candidate becomes the nominee of the Democratic party and will henceforth be called the challenger.

Stage 2-General Election

- The challenger chooses an effort level $e_{2}^{C h} \in[0,1]$ for the general election campaign.

- A signal $s_{2}^{C h} \in\{\lambda, \mu\}$ regarding the challenger's type is generated and observed by all voters.

- The type of the incumbent is common knowledge, $t^{I n c}=R$.

- All voters cast their votes for one of the candidates.

- The winner of the election is determined by the majority of votes cast.

- The elected politician implements a policy equal to her type and payoffs are determined.

\section{A. Preferences}

Candidates derive utility from winning the general election and bear costs of effort exerted during the campaigns. Specifically, we assume that utility of candidate $k$ who exerted effort levels $e_{1}^{k}$ and $e_{2}^{k}$ in stages 1 and 2, respectively, is

$$
\Pi^{k}\left(e_{1}^{k}, e_{2}^{k}\right)=\left[\begin{array}{cl}
1-e_{1}^{k}-e_{2}^{k} & \text { if } k \text { won general election } \\
-e_{1}^{k}-e_{2}^{k} & \text { otherwise }
\end{array}\right.
$$

A voter $j$ with ideal policy $z_{j} \in[0,1]$ derives utility $u\left(z_{j}, p\right)=-\left(z_{j}-p\right)^{2}$ when policy $p \in\{L, M, R\}$ is implemented. Voters are forward-looking Bayesian agents who cast their votes to maximize their expected utility given the information available to them.

\section{B. Equilibrium Concept}

To analyze the outcomes of the election game, we characterize the Perfect Bayesian Equilibria (equilibria hereafter), restricting attention to those in which all voters hold the same beliefs about candidates' types. We also focus on symmetric equilibria, in which candidates with the same type employ the same strategy at each 
stage of the game. A voter who has a strict preference for one of the candidates is assumed to necessarily vote for this candidate, while a voter who is indifferent between two candidates is assumed to randomize and vote with equal probability for both. Abstention is not allowed.

\section{Assumptions}

The signal generated by candidate $k$ 's campaign in stage $i$ depends on candidate $k$ 's effort in stage $i$, candidate $k$ 's type and an exogenous parameter $n_{i} \in R_{++}$, which captures the prominence of the election stage $i \in\{1,2\}$. Candidates exert effort to distort the signal. Absent effort, the generated signal corresponds to the type of the candidate. It is harder to distort the signal in more prominent elections. More specifically, we assume that the probability that candidate $k$ 's campaign generates liberal signal $\lambda$ if candidate $k$ exerted effort $e_{i}^{k}$ in stage $i$ in an election with prominence $n_{i}$ is

$$
\operatorname{Pr}\left[s_{i}^{k}=\lambda\right]=\left[\begin{array}{ll}
h\left(e_{i}^{k}, M, n_{i}\right) & \text { if } t^{k}=M \\
1-h\left(e_{i}^{k}, L, n_{i}\right) & \text { if } t^{k}=L .
\end{array}\right.
$$

With the remaining probability candidate $k$ 's campaign generates moderate signal $\mu$. We refer to function $h(\cdot)$ as the scrutiny function and assume that it satisfies assumptions (A1a)-(A1f):

(A1a) $h$ is twice continuously differentiable, strictly increasing and strictly concave in effort, $\frac{\partial h(e, t, n)}{\partial e} \equiv h_{e}(e, t, n)>0$ and $\frac{\partial^{2} h(e, t, n)}{\partial e^{2}} \equiv h_{e e}(e, t, n)<0$.

(A1b) $\lim _{e \rightarrow 0} h_{e}(e, t, n)=\infty$ and $\lim _{e \rightarrow 1} h_{e}(e, t, n)<1$.

(A1c) Absent effort candidate's signal coincide with her type, $h(0, t, n)=0$.

(A1d) Highest effort does not guarantee a signal opposite from candidate's type, $h(1, t, n)<1$.

(A1e) It is harder to generate a signal opposite to the candidate's type in more prominent elections, $\frac{\partial h(e, t, n)}{\partial n} \equiv h_{n}(e, t, n)<0$.

(A1f) The signal is less responsive to candidate's additional effort in more prominent elections, $\frac{\partial h_{e}(e, t, n)}{\partial n} \equiv h_{e n}(e, t, n)<0$.

In addition, we make the following assumptions on the parameters of the election game:

(A2) Candidates' locations satisfy $0<L<M<R<1$. 
(A3) The locations of registered Democrats are drawn independently from the locations of other voters and less than half of the voters are registered Democrats.

(A4) The location of the median voter is drawn from a CDF $F$, which is continuous, strictly increasing, and differentiable on the support $[\underline{m}, \bar{m}]$ with density $f(m)$, where $\bar{z}<\underline{m}<\frac{R+L}{2}$ and $\frac{R+M}{2}<\bar{m}<1$.

(A5) The location of median Democrat is drawn from a CDF $G$, which is continuous, strictly increasing, and differentiable on the support $[\underline{d}, \bar{d}]$ with density $g(d)$, where $0<\underline{d}<\bar{d}<\min \left\{\bar{z}, \frac{L+M}{2}\right\}$.

(A6) The general election is at least as prominent as the primary race, $n_{1} \leq n_{2}$.

\section{Discussion of Modeling Choices and Interpretations}

The above assumptions pin down preferences of all registered Democrats (including median Democrat) as well as location of median voter.

First, median voter is located to the right of the most conservative Democrat, $m>\bar{z}$, which means that median voter is not a registered Democrat. This conclusion follows from assumption (A3), which asserts that registered Democrats constitute less than half of the electorate, and restrictions imposed on the support of CDF of median voter $F$ by assumption (A4).

Second, assumptions (A2) and (A5) ensure that median Democrat $d$ prefers liberal type over moderate type over the incumbent,

$$
u(d, L)>u(d, M)>u(d, R) \quad \forall d \in[\underline{d}, \bar{d}] .
$$

Third, all registered Democrats prefer to see any of the Democratic candidates in office over the incumbent, which follows from assumptions (A3) and (A4)

$$
\min \left\{u\left(z_{j}, L\right), u\left(z_{j}, M\right)\right\}>u\left(z_{j}, R\right) \quad \forall z_{j} \in[0, \bar{z}] .
$$

One of the main tensions in the model comes from a mismatch between the preferences of the median Democrat who plays an important role in selecting the nominee of the Democratic party and the preferences of the general election median who determines the final winner of the general election. Moreover, since registered Democrats, just like all voters in our model, are forward-looking expected utility maximizers, they have to weigh the probability of different challengers winning the general election as well as the proximity of their types compared to their most preferred policies.

The uncertainty about median voters captures unpredictability of the turnout in both election stages and its vulnerability to various shocks, such as weather, political scandals, and the economic environment, that may shift voters' preferences before the election day. Technically, the uncertainty about general election median $m$ generates a continuous probability of winning general election function, which otherwise 
would be a step function and significantly complicate the equilibrium characterization. The uncertainty about the median Democrat $d$ is not essential for the results.

Prior to any election stage, be that the Democratic primary or the general election, candidates campaign in an attempt to influence their chances of winning. For politicians, the campaigning process is modeled as choosing a costly effort level, which represents how hard a politician tries to misrepresent her own type and mimic the other type. For voters, the campaign process generates a signal about the type of the politician, which voters use to update their beliefs. The scrutiny function translates politicians' efforts into the likelihood of generated signals through the prism of the prominence of the race. The prominence of the race represents all the factors related to the salience, informativeness, and visibility of a race such as how much time and resources voters devote to following this race, their ability to absorb campaign information, and the media coverage of the race.

Some of the assumptions imposed on the shape of the scrutiny function $h$ are technical, while others are tied to the interpretation. The later assumptions are as follows. A candidate that exerts no effort necessarily generates signal that corresponds to her type (assumption (A1c)) $\cdot{ }^{8} \mathrm{~A}$ candidate can never generate the signal opposite from her type with certainty (assumption (A1d)). The higher the effort, the higher is the likelihood of generating a signal opposite from one's true type and the marginal increase in successful mimicking is decreasing in effort (assumption (A1a)). Further, for a given effort exerted by a candidate, the higher the prominence of a race, the more likely voters are to receive a signal that matches the true type of a candidate (assumption (A1e)), and the marginal increase of the successful mimicking function is decreasing in the prominence level of the election (assumption (A1f)). ${ }^{9}$

Each election stage is characterized by its own prominence level: $n_{1}$ is the prominence of the primary election and $n_{2}$ is the prominence of the general election. Our basic model treats $n_{1}$ and $n_{2}$ as exogenous parameters and assumes that the general election race is at least as prominent as the primary race (assumption (A6)). In Section VB, we explore the model in which candidates can influence the prominence of the primary election.

Finally, our basic model considers the information structure in which all voters, irrespective of whether they participate in the primary election or not, observe signals generated by the candidates' campaigns during the primary election. In Section VA, we explore the importance of this assumption and consider a modified election game, in which voters who do not belong to the Democratic party do not observe signals from the primary election and maintain their prior belief that each Democratic nominee is equally likely to be moderate and liberal.

\footnotetext{
${ }^{8}$ The role of this assumption is discussed in online Appendix B and in the intuition presented after Claim 3.

${ }^{9}$ For instance, the scrutiny function of the following type satisfies all imposed assumptions (A1a)-(A1f):

$$
h(e, t, n)=\phi(n) \cdot e^{a} \quad \text { where } a \in(0,1) \text { and } \forall n \text { we have }\left\{\begin{array}{l}
\phi(n) \in(0,1) \\
\phi^{\prime}(n)<0
\end{array}\right. \text {. }
$$
}




\section{Pandering Equilibrium}

We solve the model using a backward induction approach. We start by introducing notation, then characterize an equilibrium we term a pandering equilibrium (Theorem 1) and discuss the intuition behind its derivation through the series of claims. The complete proofs are presented in online Appendix A.

Since we focus on equilibria in which candidates with the same type employ the same strategy at each election stage, when we describe efforts of candidates we omit candidates' names $k=A, B$ and instead use subscripts to indicate candidates' types. Thus, $\left(e_{i}^{L}, e_{i}^{M}\right)$ denote effort levels of liberal and moderate types in election stage $i \in\{1,2\}$. Moreover, we use $\left(\hat{e}_{i}^{L}, \hat{e}_{i}^{M}\right)$ to denote voters' conjectures about efforts of liberal and moderate types in stage $i \in\{1,2\}$, and $\left(e_{i}^{L^{*}}, e_{i}^{M^{*}}\right)$ to indicate the equilibrium effort levels of candidates in stage $i \in\{1,2\}$.

Voters' beliefs are summarized by the likelihood that a candidate is believed to be moderate. Since we focus on equilibria in which all voters hold the same beliefs about candidates' types at each stage of the election, these beliefs can be summarized by function $p_{i}^{k}(\cdot)$, which indicates the likelihood that candidate $k$ is believed to be moderate at the end of stage $i \in\{1,2\}$. More precisely, voters' belief regarding the type of candidate $k$ in stage $i$ is a function of signal generated by candidate $k$ 's campaign in stage $i$, voters' conjectures about effort levels of different candidates in stage $i$ and voters' belief about $k$ 's type in the previous stage

$$
p_{i}^{k} \equiv p_{i}^{k}\left(s_{i}^{k}, \hat{e}_{i}^{L}, \hat{e}_{i}^{M}, p_{i-1}^{k}\right) \text {. }
$$

Note that in stage $i$ voters do not need to remember the exact signal generated by politician's campaign in the previous stage. All necessary information is summarized by the probability that this politician is a moderate type, $p_{i-1}^{k}$. When this does not create confusion, we will abuse the notation and write $p_{i}^{k}\left(s_{i}^{k}\right)$ instead of $p_{i}^{k}\left(s_{i}^{k}, \hat{e}_{i}^{L}, \hat{e}_{i}^{M}, p_{i-1}^{k}\right)$ for $s_{i}^{k} \in\{\lambda, \mu\}$. Similarly, we will write $p_{2}^{C h}\left(s_{2}^{C h}\right)$ to indicate voters' belief about challenger's type after they received signal $s_{2}^{C h}$ from challenger's general election campaign and $p_{1}^{C h}$ to indicate voters' belief about challenger's type at the end of the primary race, which is the prior for the general election stage. ${ }^{10}$

By the Bayes' rule

$$
\begin{aligned}
& p_{i}^{k}(\mu)=\frac{p_{i-1}^{k}\left(s_{i-1}^{k}\right) \cdot\left(1-h\left(\hat{e}_{i}^{M}, M, n_{i}\right)\right)}{p_{i-1}^{k}\left(s_{i-1}^{k}\right) \cdot\left(1-h\left(\hat{e}_{i}^{M}, M, n_{i}\right)\right)+\left(1-p_{i-1}^{k}\left(s_{i-1}^{k}\right)\right) \cdot h\left(\hat{e}_{i}^{L}, L, n_{i}\right)} \\
& p_{i}^{k}(\lambda)=\frac{p_{i-1}^{k}\left(s_{i-1}^{k}\right) \cdot h\left(\hat{e}_{i}^{M}, M, n_{i}\right)}{p_{i-1}^{k}\left(s_{i-1}^{k}\right) \cdot h\left(\hat{e}_{i}^{M}, M, n_{i}\right)+\left(1-p_{i-1}^{k}\left(s_{i-1}^{k}\right)\right) \cdot 1-h\left(\hat{e}_{i}^{L}, L, n_{i}\right)} .
\end{aligned}
$$

\footnotetext{
${ }^{10}$ As the candidates can be only one of two types, $L$ or $M$, we can use the posterior about one's type from the previous stage of the election game as the prior for the next election stage.
} 
We let $E u\left(z_{j}, p_{1}^{k}\left(s_{1}^{k}\right)\right)$ denote the expected utility of the voter who has ideal point $z_{j}$ when the winner of the primary stage is a candidate $k$ who generated a signal $s_{1}^{k}$ in the primary campaign and, thus, is believed to be moderate with probability $p_{1}^{k}\left(s_{1}^{k}\right)$.

Finally, the chances of the challenger winning the general election can be summarized by a function $W\left(p_{2}^{C h}\left(s_{2}^{C h}\right)\right)$, which we refer to as the winning general election function. This function specifies the likelihood that the challenger who is believed to be moderate with probability $p_{2}^{\mathrm{Ch}}\left(s_{2}^{C h}\right) \in[0,1]$ at the end of the general election campaign wins the final vote.

CLAIM 1: Assume that the parameters of the election game satisfy assumptions (A2) and (A4). Then, the probability that the challenger wins the general election is strictly increasing in the belief she is a moderate type at the culmination of the general election campaign.

Claim 1 asserts that the higher the likelihood that the challenger is moderate, the higher are her chances of winning the election. Depending on the beliefs about challenger's type at the end of the general election campaign, $p_{2}^{\mathrm{Ch}}\left(s_{2}^{\mathrm{Ch}}\right)$, the winning general election function can be written as

$$
W\left(p_{2}^{C h}\left(s_{2}^{C h}\right)\right)=F\left[\frac{R^{2}-p_{2}^{C h}\left(s_{2}^{C h}\right) \cdot M^{2}-\left(1-p_{2}^{C h}\left(s_{2}^{C h}\right)\right) \cdot L^{2}}{2 \cdot\left(R-p_{2}^{C h}\left(s_{2}^{C h}\right) \cdot M-\left(1-p_{2}^{C h}\left(s_{2}^{C h}\right)\right) \cdot L\right)}\right],
$$

where $F$ is the CDF of the location of the median voter. Assumptions (A2) and (A4) guarantee that the argument and, thus, the winning general election function itself is strictly increasing in $p_{2}^{C h}\left(s_{2}^{C h}\right)$. Moreover,

$$
0<W(0)<W(1)<1
$$

where $W(0)$ denote the likelihood that the challenger who was revealed to be liberal for sure wins the general election and, similarly, $W(1)$ denotes the likelihood that the challenger who was revealed to be moderate for sure wins the general election. Last inequality (3) means that both liberal and moderate candidates, if their types were revealed at some stage of the election game, have a positive but less than one chance of winning the general election.

Define $(x, y) \in(0,1) \times(0,1)$ that satisfy system of equations $(4 a)$ and $(4 b)$ :

$$
\left\{\begin{array}{l}
h_{x}\left(x, M, n_{1}\right) \cdot\left[\frac{3-h\left(x, M, n_{1}\right)}{4} W\left(p_{2}^{C h}(\mu)\right)-\frac{1-h\left(x, M, n_{1}\right)}{4} W(1)\right]=1 \\
h_{y}\left(y, L, n_{2}\right) \cdot\left[W\left(p_{2}^{C h}(\mu)\right)-W(0)\right]=1,
\end{array}\right.
$$

where

$$
p_{2}^{C h}(\mu)=\frac{h\left(x, M, n_{1}\right)}{h\left(x, M, n_{1}\right)+h\left(y, L, n_{2}\right)} .
$$


Further, for any $(x, y) \in(0,1) \times(0,1)$ that satisfy system of equations $(4 \mathrm{a})$ and (4b), define the cutoff value $\bar{M}=\gamma(x, y) \cdot L+(1-\gamma(x, y)) \cdot R$, where $p_{2}^{C h}(\mu)$ is defined by equation (5), and function $\gamma:(0,1) \times(0,1) \rightarrow R$ is defined by equation (6)

$$
\gamma(x, y)=\frac{h\left(x, M, n_{1}\right) \cdot W(0)+h\left(y, L, n_{2}\right) \cdot\left[W\left(p_{2}^{C h}(\mu)\right)-W(0)\right]}{W(1)+h\left(x, M, n_{1}\right) \cdot\left[W(1)-W\left(p_{2}^{C h}(\mu)\right)\right]} .
$$

Finally, define conditions (C1) and (C2) as follows:

condition $(\mathrm{C} 1): 2 W(0)>W(1)$ and function $W(\cdot)$ is weakly concave

condition $\left.\left.(\mathrm{C} 2): E u\left(z_{i}^{*}, 0\right)\right) \geq E u\left(z_{i}^{*}, 1\right)\right)$ where $z_{i}^{*}=\left[\begin{array}{lll}0 & \text { if } \quad M \leq \bar{M} \\ \bar{d} & \text { if } \quad M>\bar{M}\end{array}\right.$.

Conditions $(\mathrm{C} 1)$ and $(\mathrm{C} 2)$ play important roles in ensuring that the majority of the registered Democrats support the candidate whose type is uncertain over the candidate who was revealed to be moderate during the primary campaign (see detailed discussion in Claim 4). To preview this discussion, condition (C1) puts an upper bound on the advantage of a revealed moderate over a revealed liberal candidate in terms of likelihood of winning the general election stage. Condition (C2) identifies which Democratic voter has the "biggest hesitation" (smallest difference in expected utility terms) when choosing between a revealed liberal and a revealed moderate in the primary election: when the moderate candidate is relatively liberal, $M \leq \bar{M}$, then it is the most liberal voter $z_{j}=0$ who "hesitates" the most; however, if the moderate candidate is relatively conservative, $M>\bar{M}$, then it is the most conservative median Democrat $\bar{d}$ who "hesitates" the most.

THEOREM 1: Assume that the parameters of the election game satisfy assumptions (A1)-(A6) and conditions $(C 1)$ and (C2). Then, there exists a Pandering Equilibrium, in which effort levels of candidates are $\left(e_{1}^{M^{*}}, e_{1}^{L^{*}}, e_{2}^{M^{*}}, e_{2}^{L^{*}}\right)$ $=(x, 0,0, y)$, where $(x, y)$ are defined above. If $s_{1}^{l}=\mu$ and $s_{1}^{k}=\lambda$, then candidate $k$ wins the primary election for sure.

Theorem 1 asserts that there exists an equilibrium in which in the primary campaign liberal politicians exert no effort, while moderate ones mimic liberal types to some extent. If one candidate generates liberal $\lambda$ signal, while another generates moderate $\mu$ signal, then the former wins the primary race for sure, becomes the nominee of the Democratic party (challenger) and advances to the general election stage. If the Democratic nominee generated moderate signal in the primary race and still won the primary, then irrespectively of her type, she puts no effort in the general election campaign. If, however, the Democratic nominee generated the liberal signal and won the primary, then her behavior in the general election depends on her type: moderate challenger puts no effort, while liberal challenger mimics moderate to some extent and puts positive amount of effort. 
In the remainder of this section, we discuss main trade-offs faced by the voters and the candidates at different stages of the election game and provide intuition for the conditions (C1) and (C2). We start by analyzing the challenger's behavior during the general election campaign (Claims 2 and 3), then discuss voters' behavior in the primary election (Claim 4) and, finally the behavior of Democratic candidates during the campaign that precedes the primary election (Claim 5).

\section{A. Challenger's Behavior in the General Election}

The challenger's behavior during the general election campaign depends on voters' belief about her type carried over from the primary election.

CLAIM 2: Assume that the parameters of the election game satisfy assumptions (A1)-(A6). If the belief about challenger's type at the beginning of the general election stage is degenerate, $p_{1}^{C h}=0$ or $p^{C h}=1$, then she exerts no effort irrespectively of her type. If, however, voters have even a small doubt about challenger's type after the primary race, i.e., $p_{1}^{C h} \in(0,1)$, then the unique equilibrium in the general election subgame prescribes the moderate challenger to exert no effort and the liberal challenger to put positive effort in mimicking the moderate type, where the amount of mimicking $e_{2}^{L^{*}}$ is determined by equation $(3 b)$ and

$$
p_{2}^{C h}(\mu)=\frac{p_{1}^{C h}}{p_{1}^{C h}+\left(1-p_{1}^{C h}\right) \cdot h\left(e_{2}^{L^{*}}, L, n_{2}\right)} .
$$

To intuit Claim 2, suppose that voters conjecture that the challenger exerts efforts $\left(\hat{e}_{2}^{L}, \hat{e}_{2}^{M}\right)$ in the general election campaign depending on her true type. Then, expected payoff of a moderate challenger if she exerts effort $e_{2}^{M}$, denoted by $E \Pi^{t^{C h}=M}\left(e_{2}^{M}\right)$, can be written as

$$
\begin{aligned}
E \Pi^{t^{C h}=M}\left(e_{2}^{M}\right)= & -e_{2}^{M}+W\left(p_{2}^{C h}(\mu)\right) \\
& -h\left(e_{2}^{M}, M, n_{2}\right) \cdot\left[W\left(p_{2}^{C h}(\mu)\right)-W\left(p_{2}^{C h}(\lambda)\right)\right] .
\end{aligned}
$$

Similarly, expected payoff of a liberal challenger if she exerts effort $e_{2}^{L}$, denoted by $E \Pi^{t^{C h}}=L\left(e_{2}^{L}\right)$, can be written as

$$
E \Pi^{t^{C h}=L}\left(e_{2}^{L}\right)=-e_{2}^{L}+W\left(p_{2}^{C h}(\lambda)\right)+h\left(e_{2}^{L}, M, n_{2}\right) \cdot\left[W\left(p_{2}^{C h}(\mu)\right)-W\left(p_{2}^{C h}(\lambda)\right)\right],
$$

where $p_{2}^{C h}(\mu)$ and $p_{2}^{C h}(\lambda)$ are determined based on the voters' believed levels of candidates' efforts $\hat{e}_{2}^{L}$ and $\hat{e}_{2}^{M}$, as specified in equations (1a) and (1b). In other words, by choosing to put in higher or lower effort, the challenger can change the likelihood of generating moderate and liberal signals but not voters' beliefs about her true type conditional on a received signal. Of course, in equilibrium, voters' beliefs are correct and $e_{2}^{k^{*}}=e_{2}^{k}=\hat{e}_{2}^{k}$ for $k \in\{L, M\}$.

The only beliefs $\left(\hat{e}_{2}^{L}, \hat{e}_{2}^{M}\right)$ that are consistent with equilibrium behavior of challenger in the general election stage are the beliefs according to which the moderate 
signal $\mu$ indicates higher chance that challenger is truly moderate than the liberal signal $\lambda$, that is, $p_{2}^{C h}(\mu)>p_{2}^{C h}(\lambda)$. This is the reason that the moderate challenger chooses zero effort, which means that a liberal signal observed during the general election campaign is perfectly informative, $p_{2}^{C h}(\lambda)=0$. The optimal amount of effort for the liberal challenger is determined by the conditions that equate marginal benefit of effort to the marginal cost:

$$
\frac{d E \Pi^{t^{C h}=L}\left(e_{2}^{L}\right)}{d e_{2}^{L}}=-1+h_{e}\left(e_{2}^{L}, L, n_{2}\right) \cdot\left[W\left(p_{2}^{C h}(\mu)\right)-W(0)\right] .
$$

The marginal cost of extra unit of effort is one, while the marginal benefit takes into account both an increase in the likelihood of generating moderate signal and a higher chance of winning the general election conditional on a moderate rather than liberal signal being generated. The assumption $h_{e}\left(0, L, n_{2}\right) \rightarrow \infty$ guarantees that a liberal challenger always mimics the moderate type to some extent. The uniqueness of optimal effort of a liberal challenger follows from the properties of the probability winning and scrutiny functions, since higher belief $\hat{e}_{2}^{L}$ reduces the marginal benefit of effort for liberal challenger.

Next we explore how an optimal amount of mimicking depends on voters' beliefs about a challenger's type carried over from the primary election, $p_{1}^{C h}$.

CLAIM 3: Assume that the parameters of the election game satisfy assumptions (A1)-(A6) and voters are uncertain about a challenger's type at the beginning of the general election stage, $p_{1}^{C h} \in(0,1)$. Then, the liberal challenger exerts more effort in pretending to be moderate during the general election campaign when prior on her being moderate at the beginning of the general election campaign is higher:

$$
\frac{d e_{2}^{L^{*}}}{d p_{1}^{C h}}>0
$$

To gain intuition for Claim 3, first note that absent any effort, either politician generates the signal associated with their type. Politicians must exert effort to obfuscate their type. As is often the case in signaling problems, both agents would like to appear to be the same type, in this case, the moderate type. So, the moderate type makes no effort obfuscating this signal and generates the moderate signal for sure. The liberal type's returns from generating the misleading moderate signal will then depend on the prior in the following way. Conditional on generating the moderate signal, the liberal type can be either moderate or a liberal type who, through effort, generated the moderate signal. The stronger the prior on the moderate type, the more weight the electorate place on the former of these possibilities and the greater the belief from generating the moderate signal. In contrast, regardless of the prior belief, liberal signal reveals that the challenger is liberal for sure. This leads the liberal type to exert more effort when the prior on her being moderate is higher. 
One of the important assumptions that Claim 3 uses is (A1c), which states that a candidate that exerts no effort generates signal corresponding to her type for sure. ${ }^{11}$ To highlight the role of this assumption, consider a modified election model, in which a candidate that exerts no effort has a small but positive chance of generating a signal opposite from her type, that is, $h(0, t, n)=\epsilon>0$. All the remaining assumptions are the same as in the basic election model. Then, the optimal effort of the liberal challenger in the general election stage is a single-peaked function, which starts and finishes at zero for the degenerate beliefs $p_{1}^{C h}=0$ and $p_{1}^{C h}=1$, respectively. However, as $\epsilon$ approaches zero, the peak of this function shifts to the right, that is, $p_{1}^{C h}$ that maximizes $e_{2}^{L^{*}}\left(p_{1}^{C h}\right)$ approaches one. In particular, for $\epsilon=0$, the optimal effort of the liberal challenger is strictly increasing in $p_{1}^{C h}$ on the domain $(0,1)$ but drops discontinuously at $p_{1}^{C h}=1$ to zero. We refer the reader to online Appendix B for the formal statement and proof of this result.

\section{B. Primary Stage}

Now that we have determined the challenger's behavior in the general election stage, we move back to the primary stage and discuss the behavior of primary voters. If posteriors about Democratic candidates at the end of the primary campaign are the same, then candidates are indistinguishable in the voters' eyes and each candidate wins the nomination with equal probability. If, however, voters choose between a moderate for sure candidate and a candidate of uncertain type, then the uncertain type wins the nomination and advances to the general election stage.

CLAIM 4: Assume that the parameters of the election game satisfy assumptions (A1)-(A6) and conditions (C1) and (C2). Then, a Democrat who is believed to be moderate for sure at the culmination of the primary campaign period loses against a Democrat whose type is uncertain.

Primary voters are forward-looking and, thus, evaluate candidates running in the primary election taking into account both candidate's ideology and her behavior in the general election campaign. All registered Democrats can be classified into two groups according to their rankings of candidates' types:

\section{Group 1 "Liberal Democratic Voters" $u\left(z_{j}, L\right)>u\left(z_{j}, M\right)>u\left(z_{j}, R\right)$ Group 2 "Conservative Democratic Voters" $u\left(z_{j}, M\right)>u\left(z_{j}, L\right)>u\left(z_{j}, R\right)$.}

The exact location of most conservative Democrat $\bar{z}$ determines the composition of the Democratic party. If $\bar{z} \leq \frac{L+M}{2}$, then the Democratic party consists of liberal Democratic voters only, while if $\bar{z} \in\left(\frac{L+M}{2}, \frac{L+R}{2}\right)$, then both liberal and conservative Democratic voters are present.

\footnotetext{
${ }^{11}$ We thank an anonymous referee for suggesting we explore this further.
} 
To see why a moderate candidate loses against an uncertain type in the primary election, we will consider the behavior of each of the groups separately. For a voter with ideal policy $z_{j} \in[0, \bar{z}]$, define by $S\left(z_{j}\right)$ the difference in expected utility between the scenario in which the Democratic nominee is a candidate $k$ who, at the end of the primary stage, is believed to by moderate with probability $p_{1}^{k} \in(0,1)$, and the scenario in which the Democratic nominee is candidate $l$ who is for sure moderate, $p_{1}^{l}=1$. This difference can be written as

$$
S\left(z_{j}\right)=\epsilon \cdot\left[u\left(z_{j}, R\right)-u\left(z_{j}, M\right)+\psi \cdot\left(u\left(z_{j}, L\right)-u\left(z_{j}, R\right)\right)\right],
$$

where $\psi=\frac{\left(1-p_{1}\right) \cdot W(0)+\frac{p_{1}\left(1-p_{2}(\mu)\right)}{p_{2}(\mu)} \cdot\left(W\left(p_{2}(\mu)\right)-W(0)\right)}{\epsilon} \in(0,1)$ and $\epsilon=W(1)-$ $p_{1} \cdot W\left(p_{2}(\mu)\right)>0$.

Conservative Democratic voters have $S\left(z_{j}\right)<0$ and, thus, will support a sure moderate over a candidate with uncertain type in the primary. ${ }^{12}$ On the contrary, liberal Democratic voters support the uncertain over the sure moderate type provided that conditions $(\mathrm{C} 1)$ and $(\mathrm{C} 2)$ hold. In addition, these conditions guarantee that liberal Democratic voters constitute a majority of voters in the Democratic party.

To understand the role of conditions ( $\mathrm{C} 1)$ and $(\mathrm{C} 2)$, consider the trade-off that liberal Democratic voters face. Just like any other voter in this game, liberal Democratic voters weigh relative importance of two factors that jointly determine their payoffs: candidates' ideology and their chances of winning the general election conditional on behaving optimally in the general election stage. In terms of ideology, liberal Democratic voters prefer a liberal over a moderate candidate, and, consequently, a candidate with an uncertain type over the moderate one. However, the latter has a higher chance of winning the general election if nominated than the former for any given level of the general election prominence $n_{2}$. The condition $(\mathrm{C} 1)$ puts a restriction on how much more likely a sure moderate is to win the general election compared with a sure liberal challenger, which bounds an advantage that the moderate type has over the liberal ones in terms of likelihood of winning the general election. The condition ( $\mathrm{C} 2)$ distinguishes between two cases according to the location of the moderate candidate. If a moderate type is ideologically "close" to a liberal type, then it is enough to ensure that the most liberal Democratic voter, $z_{j}=0$, prefers to nominate liberal over moderate type to guarantee that so do all other liberal Democratic voters. However, if a moderate type is ideologically far away from a liberal type, then as long as the most conservative median Democrat supports a liberal over a moderate type for the nomination, so will all other liberal Democratic voters.

CLAIM 5: Assume that the parameters of the election game satisfy assumptions $(A 1)-(A 6)$ and conditions $(C 1)$ and (C2). Then, there exists an equilibrium, in which a liberal politician puts no effort in the primary campaign, while a moderate politician mimics behavior of a liberal one to some extent. The optimal amount of

\footnotetext{
${ }^{12}$ Indeed, for conservative Democratic voters $\left.u\left(z_{j}\right), M\right)-u\left(z_{j}, R\right)>\psi \cdot\left(u\left(z_{j}, L\right)-u\left(z_{j}, R\right)\right)$, since $\psi \in(0,1)$. This, coupled with $\epsilon>0$, guarantees that conservative Democratic voters have $S\left(z_{j}\right)<0$.
} 
mimicking is determined by the equation $(3 a)$, and $p_{2}^{C h}(\mu)$ is determined by equation (4).

The final step in the characterization of the pandering equilibrium (Claim 5) naturally follows from the behavior of the primary voters. Indeed, winning the primary election is a necessary step in winning the general election. Since primary voters reward politicians who are closer in the ideology to the policy preferred by the median Democrat, liberal types are happy to exert no effort in the primary campaign and moderate types have an incentive to mimic liberal types by exerting positive effort.

\section{Comparative Statics of Pandering Equilibrium}

In this section we consider how optimal behavior of politicians changes in response to changes in the environment, as captured by changes in the prominence levels of the primary and the general election campaigns, $\left(n_{1}, n_{2}\right)$. We then use these comparative statics results to derive the "divisive-primary" effect.

For the purpose of deriving comparative statics results, we will introduce one more restriction on the shape of the scrutiny function:

$$
\text { condition }(\mathrm{C} 3):-h_{e e}(e, t, n) \cdot h(e, t, n) \geq h_{e}^{2}(e, t, n) \quad \forall e \in[0,1] \text {. }
$$

The condition (C3) focuses on the subset of scrutiny functions that exhibit a relatively high degree of concavity. This condition ensures that equilibrium effort levels of the candidates can be ranked according to visibility of the primary race. For instance, if $h(e, t, n)=\phi(n) \cdot e^{a}$, where $\phi(n) \in(0,1)$ and $\phi^{\prime}(n)<0$, then condition (C3) is satisfied as long as $a \in\left(0, \frac{1}{2}\right]$.

CLAIM 6: Assume that the parameters of the election game satisfy assumptions $(A 1)-(A 6)$, conditions $(C 1)-(C 3)$, and voters and candidates are playing the pandering equilibrium described in Theorem 1. Then, we expect less pandering by both types of candidates in elections with more prominent primary races. That is, moderate candidates exert less effort in the primary race, $\frac{\partial e_{1}^{M^{*}}}{\partial n_{1}}<0$, and liberal challengers exert less effort in the general election race, $\frac{\partial e_{2}^{L^{*}}}{\partial n_{1}}<0$. Moreover, voters believe the likelihood that a moderate type generates signal $\lambda$ in the primary and signal $\mu$ in the general election is lower when the primary race is more prominent.

Intuitively, higher prominence of the primary race has two effects. First, it decreases the proportion of moderate candidates who reach the general election, as voters are better at detecting and filtering moderates in the primary race. Moderate candidates react to this by putting less effort in mimicking liberals in the primary race. Second, it discourages liberal challengers from exerting effort in the general election stage since voters put lower probability on the challenger being a moderate 
type after observing a liberal signal in the more prominent primary race (see Claim 3). The first effect decreases the likelihood that a liberal signal in the primary and a moderate signal in the general election is coming from the moderate type, while the second effect works in the opposite direction. The first effect, however, dominates the second one due to only partial adjustment of the effort level on the part of liberal challengers. Therefore, voters believe that the likelihood that a moderate candidate produced a liberal signal in the primary and a moderate signal in the general election is smaller when the candidates' selection process in the primary stage is more intense.

This comparative statics result translates directly into the chances of the Democratic Party to win the general election as described in Claim 7.

CLAIM 7: “Divisive Primary Effect." Assume that the parameters of the election game satisfy assumptions (A1)-(A6), conditions $(C 1)-(C 3)$, and voters and candidates are playing the pandering equilibrium described in Theorem 1. Then, the higher the prominence of the primary election race, the lower are the chances of the Democratic Party to win the general election.

Primaries with high visibility affect the probability of a Democrat to get elected through two distinct channels. First, primaries with high visibility make it harder for a moderate candidate to obtain the nomination, since these candidates are more likely to be detected and defeated by liberals in the primary race. Second, liberal candidates that obtain the nomination are handicapped in the general election campaign because of the informative prior they carry over after the informative primaries as described by Claim 3. Both effects are detrimental to the Democratic Party, which loses overall elections more often when primaries are more visible.

Finally, Claim 8 speaks to the effect of increasing general election visibility on the equilibrium behavior of candidates and chances of the Democratic Party to win the election.

CLAIM 8: Assume that the parameters of the election game satisfy assumptions $(A 1)-(A 6)$, conditions $(C 1)-(C 3)$, and voters and candidates are playing the pandering equilibrium described in Theorem 1 . Then, in the elections with a more prominent general election race, we expect moderate candidates to put in more effort in the primary campaign, $\frac{\partial e_{1}^{M^{*}}}{\partial n_{2}}>0$, and liberal challengers to be revealed as liberals more often during the general election campaign, $\frac{d h\left(e_{2}^{L^{*}}, L, n_{2}\right)}{d n_{2}}<0$.

In the elections, in which the second (general election) stage is more visible, moderate candidates factor in that liberal challengers are going to lose more often in the second stage, because voters subject them to a higher scrutiny process. This increases the chances of moderates winning the general election vote conditional on obtaining nomination, which provides an extra incentive to invest in winning the primary. This motivates moderate candidates to put in higher effort in the primary campaign when general elections are more prominent. 


\section{Extensions of the Model}

In this section, we consider two extensions of the basic model developed above. The first extension revisits the informational structure of the model and explores the situation in which part of the general election electorate is ignorant about events that transpire during the primary election. The second extension takes a first step at exploring how primary prominence is determined and studies the game, in which candidates can affect primary visibility via costly investments.

\section{A. Information Structure}

In this extension, we explore how robust pandering equilibrium is to the changes in the information structure of the election game. In particular, we consider the situation, in which only registered Democrats, $z_{j} \in[0, \bar{z}]$, observe signals generated by candidates's campaigns in the primary. The remaining voters, $z_{j} \in(\bar{z}, 1]$, are ignorant about what has transpired during the primary and believe that any Democratic nominee has an equal chance of being a liberal or a moderate type. Besides this informational assumption, the remaining details about the election game are the same as in the basic model studied in Sections II to IV. We refer to this version of the election game as the election game with partially informative primaries. ${ }^{13}$

Define $(q, r) \in(0,1) \times(0,1)$ that satisfy system of equations $(7 \mathrm{a})$ and $(7 \mathrm{~b})$ :

$$
\left\{\begin{array}{l}
h_{q}\left(q, M, n_{1}\right) \cdot \frac{1}{2} W\left(p_{2}^{C h}(\mu)\right)=1 \\
h_{r}\left(r, L, n_{2}\right) \cdot\left[W\left(p_{2}^{C h}(\mu)\right)-W(0)\right]=1,
\end{array}\right.
$$

where

$$
p_{2}^{C h}(\mu)=\frac{1}{1+h\left(r, L, n_{2}\right)} .
$$

Conditions ( $\mathrm{C} 1)$ and $(\mathrm{C} 2)$ are defined the same way as in the basic model, where $(q, r)$ defined by the system of equations $(7 a)$ and $(7 b)$ take place of $(x, y)$ defined in Section III.

THEOREM 2: Consider the election game with partially informative primaries and assume that parameters of the game satisfy assumptions (A1)-(A6) and conditions $(C 1)$ and (C2). Then, there exists a Pandering Equilibrium, in which effort levels of candidates are

$$
\left(\tilde{e}_{1}^{M^{*}}, \tilde{e}_{1}^{L^{*}}, \tilde{e}_{2}^{M^{*}}, \tilde{e}_{2}^{L^{*}}\right)=(q, 0,0, r)
$$

where $(q, r)$ are defined by the system of equations (7a) and (7b). If $s_{1}^{l}=\mu$ and $s_{1}^{k}=\lambda$, then candidate $k$ wins the primary election for sure.

\footnotetext{
${ }^{13}$ All the proofs are presented in online Appendix C.
} 
Theorem 2 asserts that the existence of pandering equilibrium is robust to the change in the informational structure of the election game. In particular, in spite of the primaries being observed only by the subset of the electorate, moderate candidates exert positive effort during the primary campaign in order to increase chances of winning the primary, while liberal challengers exert positive effort in the general election just like in the basic election game.

CLAIM 9: Effort levels of candidates in the election game with partially informative primaries are weakly higher than those in the basic election game, that is,

$$
e_{1}^{M^{*}}<\tilde{e}_{1}^{M^{*}} \text { and } e_{2}^{L^{*}}<\tilde{e}_{2}^{L^{*}}
$$

The main reason that candidates exert higher efforts in the election game with partially informative primaries compared to the basic model is the difference in median voter's beliefs regarding the challenger's type at the beginning of the general election stage. Since the most conservative Democratic voter is not too conservative (assumption (A4)), a median voter in the general election is not a registered Democrat and, thus, she does not observe primary campaign in the election model with partially informative primaries. Therefore, the median voter believes that the challenger is equally likely to be a moderate or a liberal type, $\tilde{p}_{1}^{C h}=\frac{1}{2}$, where $\tilde{p}_{1}^{C h}$ denotes the belief of the voters with ideal points $z_{j} \in(\bar{z}, 1]$ regarding the type of the challenger at the beginning of the general election stage in the game with partially informative primaries. On the contrary, in the basic election game, the median voter observes signals generated by candidates in the primary campaigns and updates her beliefs using the Bayes' rule. In particular, a challenger who generated a liberal signal in the primary campaign is believed to be a liberal type with higher chance than a moderate type, $p_{1}^{C h}(\lambda)=\frac{h\left(e_{1}^{M^{*}}, M, n_{1}\right)}{1+h\left(e_{1}^{M^{*}}, M, n_{1}\right)}<\frac{1}{2}$, while a challenger who generated a moderate signal in the primary campaign is believed to be moderate for sure, $p_{1}^{C h}(\mu)=1$. Since liberal challengers exert higher efforts in the general election when their prior is higher (Claim 3), the liberal challenger will put in higher effort in the game with partially informative primaries than in the basic game, $e_{2}^{L^{*}}<\tilde{e}_{2}^{L^{*}}$. Moreover, since higher belief about a challenger's type has a direct effect on the likelihood of winning the general election for the moderate candidates, $\frac{d p_{2}^{C h}(\mu)}{d p_{1}^{C h}}>0$ and $W^{\prime}\left(p_{2}^{C h}\right)>0$, moderate candidates are incentivized to put in higher effort levels in the primary election in order to increase their chances of winning the primary and advancing to the general election stage, that is, $e_{1}^{M^{*}}<\tilde{e}_{1}^{M^{*}}$.

CLAIM 10: The chances of a Democrat winning the election are higher in the game with partially informative primaries than in the basic game.

Several effects contribute to this result. First, moderate candidates win primary elections more often when primaries are partially informative, and this increases the chances of the Democratic party winning the general election as moderate 
challengers win the second stage more often than liberal challengers. Second, the median voter has higher belief about the challenger's type at the beginning of the general election stage when primaries are partially informative, compared with the basic model when a challenger generated a liberal signal during the primary campaign. This, in turn, increases the chances of winning the second stage for both moderate and liberal challengers who generate a moderate signal in the general election stage. Third, liberal challengers put in higher effort in the general election stage, and thus, increase even further their chances of winning the general election. Finally, the challenger who was revealed to be moderate in the primary and still won the nomination has a lower chance of winning the general election when primaries are partially informative. This happens because the information revealed during the primary never gets to the median voter who is not registered Democrat. However, the first three positive effects outweigh the last negative effect, and, overall the chances of a Democrat winning the general election are higher when primaries are only partially informative.

\section{B. Endogenous Primary Prominence}

While some characteristics of the political competition are less likely to be influenced by candidates' behavior (such as the capacity of voters to absorb information), there are other characteristics that are surely manipulable by candidates. For instance, candidates can spend financial and political resources to advertise the importance of a particular race through media outlets, and by this, affect how much time and attention voters devote to a particular race, which, in turn, will affect the prominence of the race. To explore these strategic considerations, we consider a modified election game, in which candidates can influence primary visibility via costly investment that precedes the primary race. This investment stage happens after candidates learn their types but before the primary campaign begins.

Specifically, after nature determines the types of candidates, each Democratic candidate privately and simultaneously chooses whether to invest an amount $c>0$ to increase primary visibility from level $\underline{n}_{1}$ to $\bar{n}_{1}$, where $\underline{n}_{1}<\bar{n}_{1} \leq n_{2}$. These investments map probabilistically into the actual prominence level of the primary race: the probability that primary prominence is $\bar{n}_{1}$ is higher when both candidates invest, intermediate if only one candidate invests, and the smallest when no candidate invests. To abstract away from additional signaling channel that such investments provide to candidates, we maintain the assumption that for any realized level of primary prominence, voters believe that each candidate is equally likely to be a moderate or a liberal type and each Democratic candidate holds the same beliefs about her opponent in the primary race. The remaining details of the election game are the same as in the basic model studied in Sections II to IV. We will refer to this version of the election game as the election game with endogenous primary prominence. 14

\footnotetext{
${ }^{14}$ All the proofs are presented in online Appendix D.
} 
THEOREM 3: Consider the election game with endogenous primary prominence and assume that parameters of the game satisfy assumptions (A1)-(A6) and conditions $(C 1)-(C 3)$. Then for $c<\bar{c}$ this game has a Pandering Equilibrium with Separation in the Investment Stage, in which liberal candidates invest in increasing primary visibility, while moderate candidates refrain from doing so. Once the primary prominence is determined based on investment decisions of candidates, $n_{1} \in\left\{\underline{n}_{1}, \bar{n}_{1}\right\}$, both candidates and voters play the Pandering Equilibrium described in Theorem 1 for a given $n_{1}$.

To prove Theorem 3, it is enough to show that expected payoff of a liberal candidate increases in primary visibility, and expected payoff of a moderate candidate decreases in primary visibility. Expected payoff of a liberal candidate, $E \Pi^{t^{k}=L}$, when both voters and candidates play pandering equilibrium given prominence level of the primary race $n_{1}$, can be written as

$$
\begin{aligned}
E \Pi^{t^{k}=L}=\frac{3-h\left(e_{1}^{M^{*}}, M, n_{1}\right)}{4} \cdot & {\left[-e_{2}^{L^{*}}+W(0)+h\left(e_{2}^{L^{*}}, L, n_{2}\right)\right.} \\
& \left.\cdot\left[W\left(\frac{h\left(e_{1}^{M^{*}}, M, n_{1}\right)}{h\left(e_{2}^{L^{*}}, L, n_{2}\right)+h\left(e_{1}^{M^{*}}, M, n_{1}\right)}\right)-W(0)\right]\right],
\end{aligned}
$$

where the first fraction represents the probability that liberal candidate wins the nomination and the expression in the brackets represents the expected payoff of a liberal candidate conditional on advancing to the general election stage and behaving as pandering equilibrium prescribes.

Higher primary visibility defers moderate candidates from exerting high effort in the primary campaign, which increases the chances of a liberal candidate winning the nomination. This increases the expected payoff of a liberal candidate. At the same time, low effort of moderate candidates lowers voters' belief about a liberal challenger that is carried over to the general election stage. The liberal challengers react to this reduction in beliefs by lowering their effort in the general election campaign. As a result, expected payoff of a liberal challenger conditional on winning the nomination is smaller when the primary is more prominent. However, the first (positive) effect outweighs the second (negative) effect and overall expected payoff of a liberal candidate increases with primary visibility.

On the contrary, moderate candidates suffer from an increase in primary visibility. Expected payoff of a moderate candidate, $E \Pi^{t^{k}=M}$, when both voters and candidates play pandering equilibrium for a given $n_{1}$, can be written as

$$
\begin{aligned}
E \Pi^{t^{j}=M}= & -e_{1}^{M^{*}}+\frac{h\left(e_{1}^{M^{*}}, M, n_{1}\right)\left(3-h\left(e_{1}^{M^{*}}, M, n_{1}\right)\right)}{4} \\
& \cdot W\left(\frac{h\left(e_{1}^{M^{*}}, M, n_{1}\right)}{h\left(e_{2}^{L^{*}}, L, n_{2}\right)+h\left(e_{1}^{M^{*}}, M, n_{1}\right)}\right)+\frac{\left(1-h\left(e_{1}^{M^{*}}, M, n_{1}\right)\right)^{2}}{4} \cdot W(1),
\end{aligned}
$$


where the first term is the cost of exerting equilibrium effort level $e_{1}^{M^{*}}$; the second term is expected payoff of the moderate candidate conditional on generating liberal signal $\lambda$ during the primary; and the third term is the expected payoff of a moderate candidate conditional on generating moderate signal $\mu$ during the primary. Recall that primary voters elect an uncertain type over the sure moderate type, which means that if signals generated by the candidates are different, then the winner of the primary is the candidate that generated a liberal signal. This means that the only possible scenario in which a candidate who generated moderate signal in the primary advances to the the general election stage is the situation in which both candidates were revealed to be moderate during the primary campaign (which is captured by the third term in $E \Pi^{t^{k}=M}$ ). While higher primary prominence decreases the effort of a moderate candidate during the primary, and, consequently, increases the first and the third term, the main effect comes from the second term, according to which moderate candidates are more likely to lose the primary, and conditional on winning the primary after generating liberal signal, they are more likely to lose the general election (see Claim 6).

To sum up, it is a liberal candidate who enjoys higher prominence of the primary race, while a moderate one prefers to minimize it.

\section{Conclusions}

In this paper, we develop a signaling model of two-stage elections in which candidates must obtain their party's nomination before competing in the general election. Candidates can choose different campaign platforms in every stage of the election, and a candidate who misrepresents his true type and wins the election incurs costs (of lying). We allow different stages of the election to have different prominence or visibility levels and demonstrate that these play an important role in the selection process of candidates.

This model provides a unified framework that allows us to examine two commonly observed patterns about primaries: the "post-primary moderation effect," in which candidates pander to the party base during the primary and shift to the center once the nomination is secured; and the "divisive-primary effect," which refers to the detrimental effect of hard-fought and prominent primaries on a party's general-election prospects.

We finish by noting that the timing of information revelation is important in twostage elections, as it affects who gets elected and which policies are implemented. While prominent primaries allow primary voters to make a more informative choice, they are also dangerous for the party in the sense that they reveal too much information about their candidates too early, and this then hurts the party's chances of winning general elections.

\section{REFERENCES}

-Abramowitz, Alan I. 1988. "Explaining Senate Election Outcomes." American Political Science Review 82 (2): 385-403.

Adams, James, and Samuel Merrill, III. 2008. "Candidate and Party Strategies in Two-Stage Elections Beginning with a Primary.” American Journal of Political Science 52 (2): 344-59. 
Alesina, Alberto. 1988. "Credibility and Policy Convergence in a Two-Party System with Rational Voters." American Economic Review 78 (4): 796-805.

Alesina, Alberto F., and Richard Holden. 2008. "Ambiguity and Extremism in Elections." Harvard Institute of Economic Research Discussion Paper 2157.

-Ali, S. Nageeb, and Navin Kartik. 2012. "Herding with collective preferences." Economic Theory 51 (3): 601-26.

Alvarez, Michael R., David T. Canon, and Patrick Sellers. 1995. "The Impact of Primaries on General Election Outcomes in the U.S. House and Senate." California Institute of Technology Division of the Humanities and Social Sciences Working Paper 932.

-Atkeson, Lonna Rae. 1998. "Divisive Primaries and General Election Outcomes: Another Look at Presidential Campaigns." American Journal of Political Science 42 (1): 256-71.

-Banks, Jeffrey S. 1990. "A model of electoral competition with incomplete information." Journal of Economic Theory 50 (2): 309-25.

- Battaglini, Marco. 2005. "Sequential voting with abstention." Games and Economic Behavior 51 (2): 445-63.

Bernstein, Robert A. 1977. "Divisive Primaries Do Hurt: U.S. Senate Races, 1956-1972.” American Political Science Review 71 (2): 540-45.

Born, Richard. 1981. "The Influence of House Primary Election Divisiveness on General Election Margins, 1962-76." Journal of Politics 43 (3): 640-61.

Burden, Barry C. 2001. "The Polarizing Effects of Congressional Primaries." In Congressional Primaries and the Politics of Representation, edited by Peter F. Galderisi, Marni Ezra, and Michael Lyons, 95-115. New York: Rowman and Littlefield Publishers.

-Callander, Steven. 2007. "Bandwagons and Momentum in Sequential Voting." Review of Economic Studies 74 (3): 653-84.

-Callander, Steven, and Simon Wilkie. 2007. "Lies, damned lies, and political campaigns." Games and Economic Behavior 60 (2): 262-86.

Calvert, Randall L. 1985. "Robustness of the Multidimensional Voting Model: Candidate Motivations, Uncertainty, and Convergence.” American Journal of Political Science 29 (1): 69-95.

Coleman, James S. 1972. "The Positions of Political Parties in Elections." In Probability Models of Collective Decision Making, edited by Richard G. Niemi and Herbert F. Weisberg, 332-57. Columbus: Charles E. Merrill Publishing Company.

Dekel, Eddie, and Michele Piccione. 2000. "Sequential Voting Procedures in Symmetric Binary Elections." Journal of Political Economy 108 (1): 34-55.

Dekel, Eddie, and Michele Piccione. 2014. "The Strategic Dis/advantage of Voting Early." American Economic Journal: Microeconomics 6 (4): 162-79.

Downs, Anthony. 1957. An Economic Theory of Democracy. New York: Harper and Row.

-Fudenberg, Drew, and Jean Tirole. 1986. "A 'signal-jamming' theory of predation.” RAND Journal of Economics 17 (3): 366-76.

Hirano, Shigeo, James M. Snyder, Jr., and Michael M. Ting. 2009. "Distributive Politics with Primaries." Journal of Politics 71 (4): 1467-80.

-Hogan, Robert E. 2003. "The Effects Of Primary Divisiveness On General Election Outcomes In State Legislative Elections." American Politics Research 31 (1): 27-47.

-Hotelling, Harold. 1929. "Stability in Competition.” Economic Journal 39 (153): 41-57.

-Hummel, Patrick. 2010. "Flip-flopping from primaries to general elections." Journal of Public Economics 94 (11): 1020-27.

-Kartik, Navin, and R. Preston McAfee. 2007. "Signaling Character in Electoral Competition." American Economic Review 97 (3): 852-70.

Kartik, Navin, and Richard Van Weelden. 2015. "Informative Cheap Talk in Elections." http://www. columbia.edu/ nk2339/Papers/KvW-communication.pdf.

Kenney, Patrick J. 1988. "Sorting out the Effects of Primary Divisiveness in Congressional and Senatorial Elections." Western Political Quarterly 41 (4): 756-77.

Key, Valdimer Orlando, Jr. 1952. Politics, Parties and Pressure Groups. 3rd ed. New York: Thomas Y. Crowell Company.

Lengle, James I., Diana Owen, and Molly W. Sonner. 1995. "Divisive Nominating Mechanisms and Democratic Party Electoral Prospects.” Journal of Politics 57 (2): 370-83.

-Meirowitz, Adam. 2005. "Informational Party Primaries and Strategic Ambiguity." Journal of Theoretical Politics 17 (1): 107-36.

Meirowitz, Adam, and Kenneth W. Shotts. 2009. "Pivots versus signals in elections." Journal of Economic Theory 144 (2): 744-71. 
Owen, Guillermo, and Bernard Grofman. 2006. "Two-stage electoral competition in two-party contests: Persistent divergence of party positions." Social Choice and Welfare 26 (3): 547-69.

Piketty, Thomas. 2000. "Voting as Communicating." Review of Economic Studies 67 (1): 169-91.

- Razin, Ronny. 2003. "Signaling and Election Motivations in a Voting Model with Common Values and Responsive Candidates." Econometrica 71 (4): 1083-19.

-Shotts, Kenneth W. 2006. "A Signaling Model of Repeated Elections.” Social Choice Welfare 27 (2): 251-61.

Westlye, Mark C. 1991. Senate Elections and Campaign Intensity. Baltimore: Johns Hopkins University Press.

-Wittman, Donald. 1983. "Candidate Motivation: A Synthesis of Alternative Theories." American Political Science Review 77 (1): 142-57. 